\title{
ALTERNATIVA PARA O APROVEITAMENTO DE SOLUÇÕES SULFOCÁUSTICAS RESIDUAIS NO PROCESSO DE POLPAÇÃO KRAFT
}

\author{
V. M. V. CRUZ ${ }^{1}$, M. S. de SOUZA ${ }^{1}$, G. L. PENA ${ }^{1}$, J. L. da $\operatorname{SILVA}^{1}$ e R. N. RIBEIRO${ }^{1}$ \\ ${ }^{1}$ Centro Universitário do Leste de Minas Gerais, Departamento de Engenharia Química \\ E-mail para contato: mellyssa95@gmail.com
}

\begin{abstract}
RESUMO - Este trabalho estudou a possibilidade de aproveitar uma solução sulfocáustica residual, proveniente da dessulfurização do GLP, no processo Kraft de polpação da celulose, objetivando a reposição dos químicos perdidos $(\mathrm{NaOH}$ e $\mathrm{Na}_{2} \mathrm{~S}$ ) durante o processo, reduzindo a necessidade de aquisição dos mesmos. Amostras de licor branco, negro e verde e do resíduo sulfocáustico foram analisadas. Realizaram-se análises para a determinação de álcali ativo, efetivo e total, teor de sais de sódio, sulfididade, teor de sulfato de sódio, teor de sólidos totais, sólidos suspensos, e viscosidade. Através do coeficiente de variação de Pearson, determinou-se a dispersão entre as características do resíduo sulfocáustico e cada um dos licores. De acordo com os dados experimentais obtidos e os resultados da determinação estatística, estimou-se que a etapa mais adequada para a inserção do resíduo sulfocáustico no ciclo de recuperação química do licor do processo Kraft seja no estágio que antecede à operação de evaporação, no tanque de licor negro fraco, para que possa ser concentrado e em seguida ter seus compostos orgânicos queimados na caldeira de recuperação.
\end{abstract}

\section{INTRODUÇÃO}

O Gás Liquefeito de Petróleo é submetido a tratamentos para remoção de poluentes tóxicos e corrosivos contidos na sua corrente. Um exemplo é o tratamento cáustico, que consiste em fazer passar a corrente de gás, contendo os compostos sulfurosos, por uma coluna de absorção recheada, sendo o recheio uma solução cáustica estagnada. Neste processo é gerada uma solução residual, com grande quantidade de $\mathrm{NaOH}$ e $\mathrm{Na}_{2} \mathrm{~S}$ (Ketu, 2013).

Por sua vez, no processo Kraft há uma demanda de químicos muito similar a este resíduo gerado no processo de dessulfurização. O licor de cozimento dos cavacos $(\mathrm{NaOH}$ e $\mathrm{Na}_{2} \mathrm{~S}$ ) é impregnado com a lignina contida na celulose após o cozimento, originando o licor negro fraco. Este é concentrado em evaporadores, gerando o licor negro forte. Após ser queimado nas caldeiras de recuperação, é gerado o smelt. Sua subsequente dissolução em água origina o licor verde, que volta a ser licor branco após a caustificação. A recuperação química dos licores é eficiente, porém, é impossível a regeneração total dos químicos. Assim, parte necessita ser reposta a cada ciclo do processo. A aquisição destes químicos responde por 23\% dos custos de produção de celulose no Brasil (DEPEC, 2016; Figueirêdo, 2009).

O presente trabalho propõe o aproveitamento de soluções sulfocáusticas residuais da dessulfurização do GLP no processo Kraft, objetivando a reposição dos químicos perdidos $\left(\mathrm{NaOH}\right.$ e $\left.\mathrm{Na}_{2} \mathrm{~S}\right)$ durante o processo. Caracterizaram-se amostras de licor branco, negro e verde, e de resíduo sulfocáustico através de ensaios químicos e físicos a fim de propor a melhor etapa do ciclo de recuperação química dos licores para a incorporação do resíduo. 


\section{MATERIAIS E MÉTODOS}

As amostras dos licores do processo Kraft foram cedidas por uma empresa produtora de polpa celulósica localizada no município de Belo Oriente/MG. As amostras da solução sulfocáustica residual, oriunda da lavagem cáustica do GLP, foram cedidas por uma petroquímica localizada no município de Contagem/MG, e estavam na metade do seu ciclo de vida. As análises realizaram-se no laboratório de química da empresa produtora de polpa celulósica cedente das amostras e nos laboratórios de química do Unileste. As amostras citadas estão presentes na Figura 1, e no Quadro 1 estão presentes as análises realizadas, bem como a metodologia utilizada. Todas as análises foram realizadas em triplicata.

Figura 1 - Amostras analisadas. a) Licores Kraft. b) Resíduo Sulfocáustico.

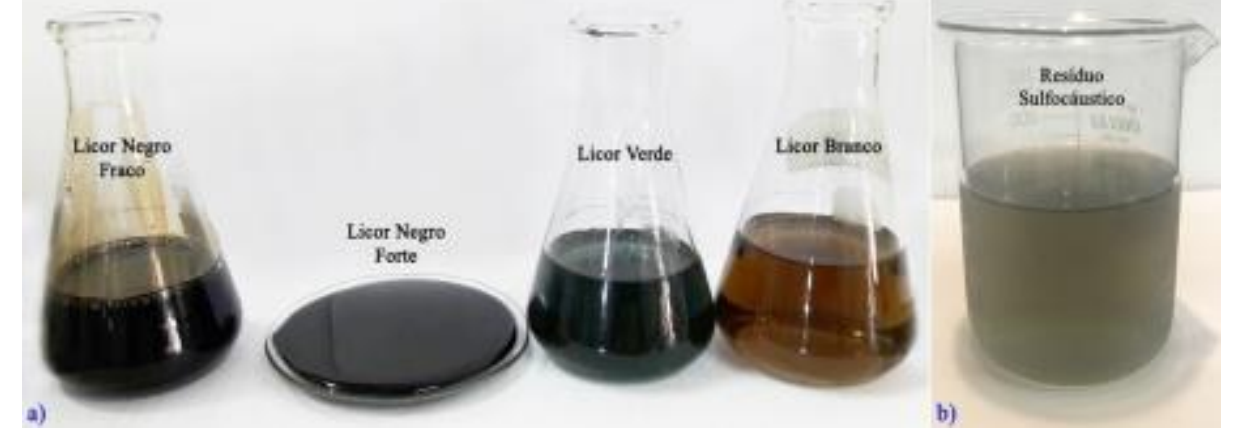

Quadro 1 - Métodos utilizados para caracterização das amostras e referências.

\begin{tabular}{|c|c|c|}
\hline Determinação & Método & Referências \\
\hline Teor de sais de sódio & Titulação com 3 pontos de inflexão & SCAN-N 2:88 \\
\hline Álcali efetivo, ativo e total & Titulação com 3 pontos de inflexão & SCAN-N 2:88 \\
\hline Sulfididade & $\begin{array}{c}\text { Razão entre conteúdo de Na } \mathrm{S} \text { e o álcali } \\
\text { ativo (LN) ou total (LV) }\end{array}$ & SCAN-N 2:88 \\
\hline Teor de sulfato de sódio $\left(\mathrm{Na}_{2} \mathrm{SO}_{4}\right)$ & Titulação por excesso de BaCl 2 & TAPPI T 624 cm-84 \\
\hline Teor de sólidos totais & $\begin{array}{c}\text { Secagem da amostra, diluída, em estufa, a } \\
\text { temperatura controlada }\end{array}$ & SCAN-N 22:96 \\
\hline Teor de sólidos suspensos & Gravimetria & TAPPI T 692 om-93 \\
\hline Viscosidade & $\begin{array}{c}\text { Viscosímetro rotacional } \\
\text { Viscosímetro do tipo capilar }\end{array}$ & $\begin{array}{c}\text { NBR 15184:2004 } \\
\text { NBR 7730:1998 }\end{array}$ \\
\hline Dispersão entre as amostras & Coeficiente de variação de Pearson $(\mathrm{CV})$ & Correa, 2003; Lopes, 2003 \\
\hline
\end{tabular}

\section{RESULTADOS E DISCUSSÃO}

Determinar o teor de sais de sódio $\left(\mathrm{NaOH}, \mathrm{Na}_{2} \mathrm{~S}\right.$ e $\left.\mathrm{Na}_{2} \mathrm{CO}_{3}\right)$ é importante, pois possibilita quantificar os químicos perdidos ao longo do processo de polpação, que precisam ser repostos. Na caldeira de recuperação, adiciona-se $\mathrm{Na}_{2} \mathrm{SO}_{4}$ ao licor negro, para reagir com a matéria orgânica presente, produzindo $\mathrm{CO}_{2}$ e $\mathrm{Na}_{2} \mathrm{~S}$. Assim, o teor de $\mathrm{Na}_{2} \mathrm{SO}_{4}$ também é um parâmetro importante de caracterização, por ser o $\mathrm{Na}_{2} \mathrm{~S}$ um dos agentes ativos no licor de cozimento (Klock et al., 2013). Na Figura 2 estão presentes os valores médios e desvio padrão obtidos para o teor de sais de sódio tituláveis (a) e para o sulfato de sódio (b) das amostras.

A maior concentração de $\mathrm{NaOH}$ encontrada foi para o licor branco, de 93,05 $\pm 3,41 \mathrm{~g} / \mathrm{L}$, seguido pelo resíduo sulfocáustico, 68,47 $\pm 0,72 \mathrm{~g} / \mathrm{L}$, pelo licor negro, 9,90 \pm 4,67 g/L, e pelo licor verde, $5,73 \pm 0,43 \mathrm{~g} / \mathrm{L}$. O Na2S está presente em maior quantidade no licor verde, 37,72 $\pm 1,10 \mathrm{~g} / \mathrm{L}$, em seguida está o licor branco, com 34,52 $\pm 2,20 \mathrm{~g} / \mathrm{L}$, o resíduo sulfocáustico, $25,97 \pm 1,54 \mathrm{~g} / \mathrm{L}$, e o licor negro, $5,12 \pm 2,83 \mathrm{~g} / \mathrm{L}$. O licor verde apresenta em maior quantidade o $\mathrm{Na}_{2} \mathrm{CO}_{3}, 107,04 \pm 2,59 \mathrm{~g} / \mathrm{L}$, seguido pelo licor branco, 19,00 \pm 1,69 g/L, resíduo 
sulfocáustico, 6,95 \pm 0,55 g/L, e licor negro, 5,71 \pm 0,68 g/L. Klock et al. (2013) afirmam que no licor verde predomina o conteúdo de $\mathrm{Na}_{2} \mathrm{~S}$ e $\mathrm{Na}_{2} \mathrm{CO}_{3}$, conforme resultados obtidos.

Figura 2 - Concentrações médias e desvio padrão. a) Sais de sódio. b) Sulfato de sódio.
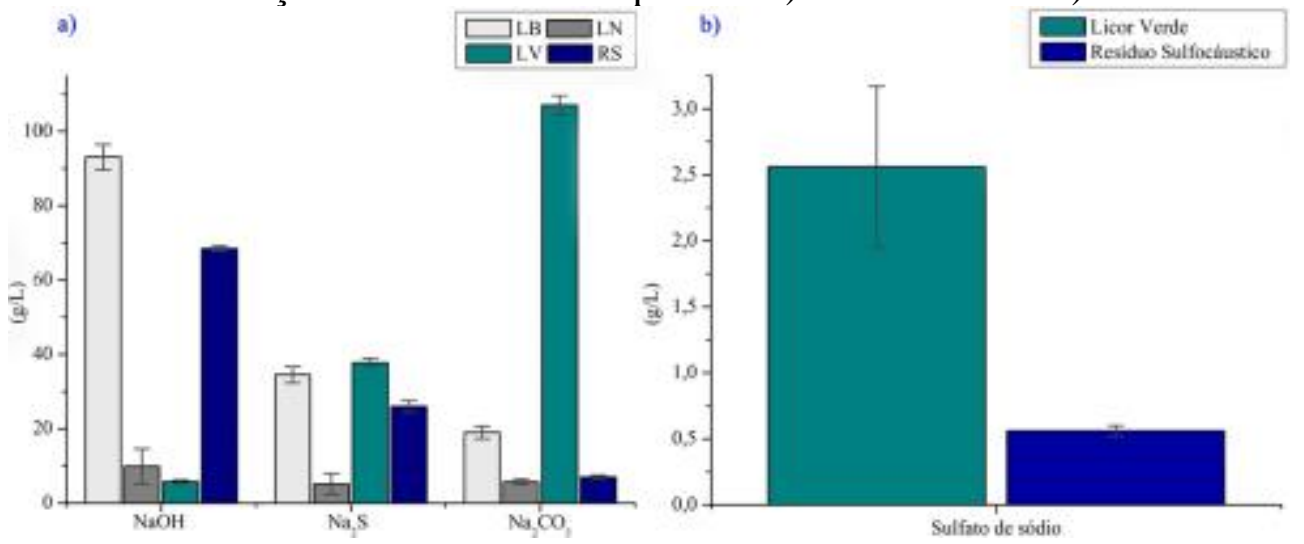

Sixta et al. (2006) citaram como composição típica do licor branco, 90 g/L de $\mathrm{NaOH}$, $40 \mathrm{~g} / \mathrm{L}$ de $\mathrm{Na}_{2} \mathrm{~S}$ e $19,8 \mathrm{~g} / \mathrm{L}$ de $\mathrm{Na}_{2} \mathrm{CO}_{3}$, estando os valores experimentais em concordância com os dados teóricos. Observa-se ainda quantidade significativa de $\mathrm{NaOH}$ e $\mathrm{Na}_{2} \mathrm{~S}$ no resíduo sulfocáustico, conforme a composição teórica citada por Ketu (2013). Em relação ao sulfato de sódio, o resíduo sulfocáustico apresentou concentração igual a 0,56 $\pm 0,04 \mathrm{~g} / \mathrm{L}$, um valor quase cinco vezes menor que o valor obtido para o licor verde, de 2,56 $\pm 0,61 \mathrm{~g} / \mathrm{L}$.

A concentração de produtos químicos ativos no processo Kraft é expressa em termos de Álcalis. Esses parâmetros são considerados os mais importantes para a caracterização dos licores. A sulfididade também é um indicativo importante, pois o $\mathrm{Na}_{2} \mathrm{~S}$ contribui para a remoção da lignina no processo de polpação. $\mathrm{O}$ pH do processo é outro fator determinante, sendo a eficiência do processo dependente deste (Klock et al., 2013; Sixta et al., 2006). Na Figura 3 estão presentes os valores médios e desvio padrão obtidos para os álcalis efetivo, ativo e total (a), bem como para a sulfididade e o $\mathrm{pH}$ (b) das amostras.

Figura 3 - Concentrações médias e desvio padrão. a) Álcalis. b) Sulfididade e pH.
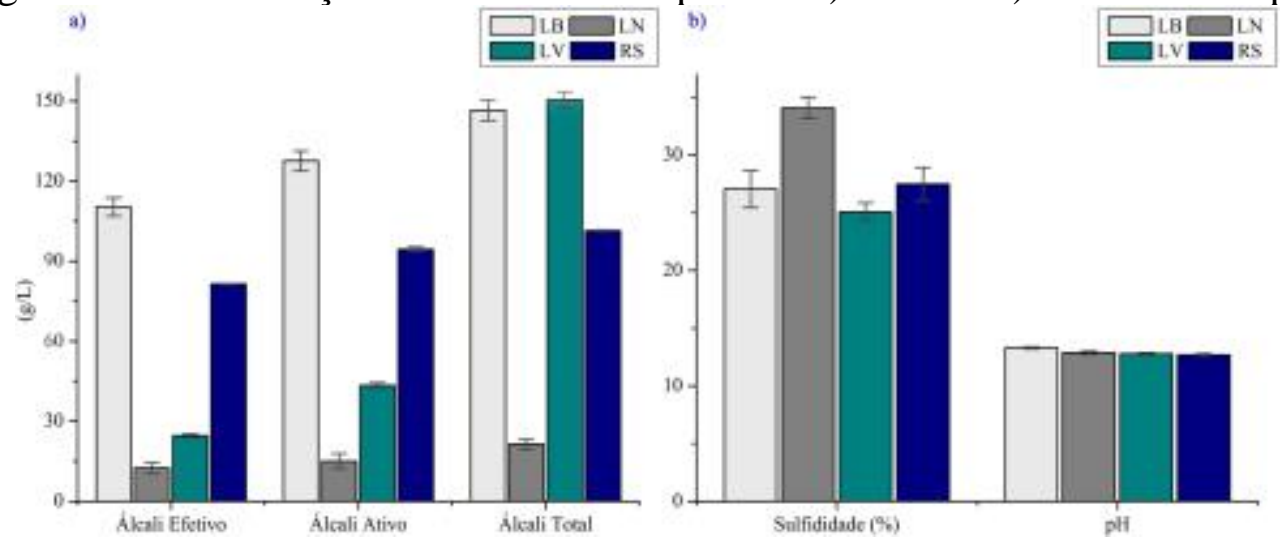

$\mathrm{O}$ álcali efetivo é a concentração total de $\mathrm{NaOH}$ e $1 / 2 \mathrm{Na}_{2} \mathrm{~S}$. O licor branco apresentou o maior valor, de $110,31 \pm 3,40 \mathrm{~g} / \mathrm{L}$, seguido pelo resíduo sulfocáustico, 81,46 $\pm 0,06 \mathrm{~g} / \mathrm{L}$, pelo licor verde, $24,58 \pm 0,56 \mathrm{~g} / \mathrm{L}$, e pelo licor negro, $12,46 \pm 2,13 \mathrm{~g} / \mathrm{L}$. O álcali ativo é a concentração total dos componentes alcalinos $\left(\mathrm{NaOH}\right.$ e $\left.\mathrm{Na}_{2} \mathrm{~S}\right)$, exceto carbonatos. O maior valor obtido foi para o licor branco, de 127,57 $\pm 3,73 \mathrm{~g} / \mathrm{L}$, seguido pelo resíduo sulfocáustico, $94,45 \pm 0,83 \mathrm{~g} / \mathrm{L}$, licor verde, 43,44 $\pm 1,02 \mathrm{~g} / \mathrm{L}$, e licor negro, 15,02 $\pm 2,86 \mathrm{~g} / \mathrm{L}$. 
$\mathrm{O}$ álcali total compreende todos os componentes alcalinos tituláveis, incluindo $\mathrm{NaOH}$, $\mathrm{Na}_{2} \mathrm{~S}, \mathrm{Na}_{2} \mathrm{CO}_{3}$. O maior valor encontrado foi para o licor verde, de 150,49 $\pm 2,92 \mathrm{~g} / \mathrm{L}$, seguido pelo licor branco, com 146,57 $\pm 3,90 \mathrm{~g} / \mathrm{L}$. O resíduo sulfocáustico apresentou 101,40 $\pm 0,28$ $\mathrm{g} / \mathrm{L}$, e o licor negro apresentou o menor valor, de $21,43 \pm 1,95 \mathrm{~g} / \mathrm{L}$, confirmando assim a grande perda dos componentes alcalinos do licor durante o processo de cozimento.

O licor negro fraco gerado no processo de cozimento é enviado a um conjunto de evaporadores, que objetiva elevar sua concentração de sólidos totais, tornando-o o licor negro forte. Esta evaporação é necessária para que o licor entre na caldeira de recuperação em condições que possibilitem sua combustão, objetivando a geração de energia elétrica para a fábrica, vapor para o processo, e a consequente formação do smelt (Klock et al., 2013).

No processo Kraft, a quantificação de sólidos suspensos é importante para as etapas de clarificação do licor verde e do licor branco, gerando um licor de cozimento de clareza adequada (Alves et al., 2015). A viscosidade é definida como a taxa de deformação do fluido, ou ainda como a resistência ao escoamento. Através de sua determinação, é possível prever o comportamento dos licores nas tubulações e nas caldeiras de recuperação do processo Kraft (Cardoso et al., 2006). Na Figura 4 estão presentes os valores médios e desvio padrão obtidos para o teor de sólidos totais e de sólidos suspensos (a), e para a viscosidade (b) das amostras.

Figura 4 - Concentração média e desvio padrão. a) Viscosidade. b) Sólidos totais e suspensos.
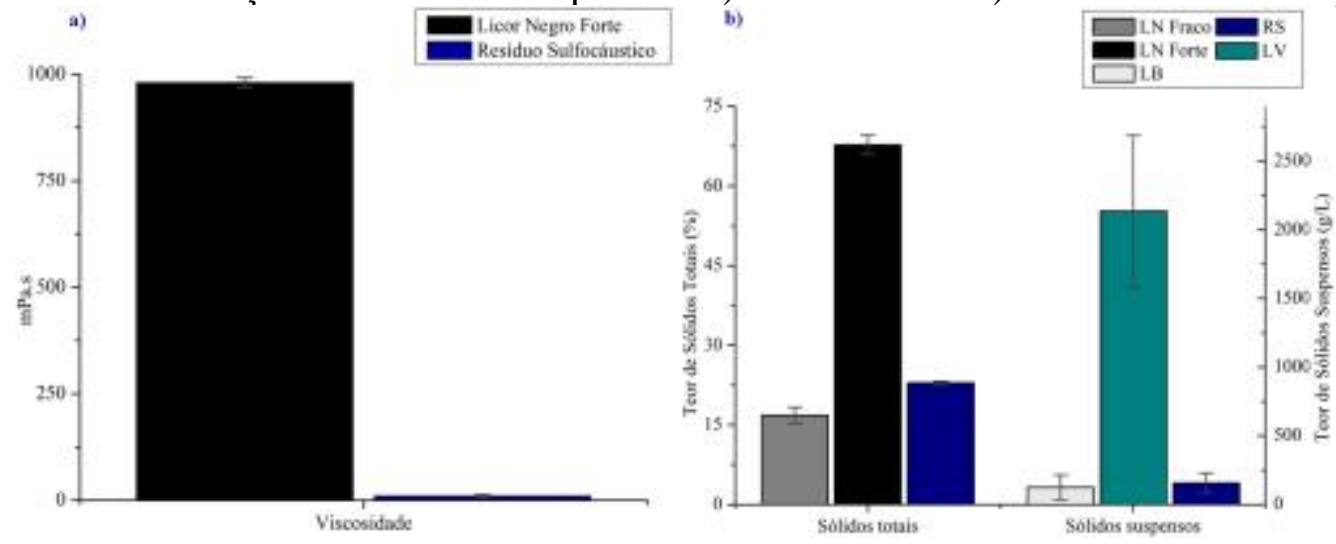

O licor negro fraco apresenta teor de sólidos totais entre 13-17\%, e o licor negro forte entre 62-73\% (Cardoso et al., 2006). O licor negro fraco apresentou 16,81 $\pm 1,53 \%$ e, após passar pela etapa de evaporação, concentrou-se para $67,76 \pm 1,81 \%$, estando esses valores na faixa teórica citada. O resíduo sulfocáustico apresentou 22,9 $\pm 0,31 \%$ de sólidos totais.

Em relação ao teor de sólidos suspensos, o licor branco apresentou 124,18 \pm 90,91 mg/L, sendo o conteúdo adequado, de acordo com Alves et al. (2015), de 50 a $100 \mathrm{mg} / \mathrm{L}$. No licor verde o conteúdo de sólidos foi de 2.138,62 $\pm 550,34 \mathrm{mg} / \mathrm{L}$. De acordo com Figueirêdo (2009), os dregs presentes no licor verde devem ser removidos, pois podem atrapalhar a etapa de caustificação. O resíduo sulfocáustico apresentou 156,00 $\pm 73,00 \mathrm{mg} / \mathrm{L}$.

O licor negro forte apresentou um valor para viscosidade de 980,00 $\pm 220,00 \mathrm{mPa}$.s. A uma temperatura de $100^{\circ} \mathrm{C}$, a viscosidade do licor negro forte pode variar de 500 a 1.000 mPa.s (Isenmann, 2012), estando o valor obtido contido na faixa citada. Para o resíduo sulfocáustico, a $20^{\circ} \mathrm{C}$, obteve-se 1,66 $\pm 0,02 \mathrm{mPa}$.s. Este valor é coerente com seu moderado teor de sólidos totais, o que configura em geral, valores mais baixos de viscosidade. 
Obtidos os dados experimentais, determinou-se estatisticamente o quanto as características do resíduo sulfocáustico se dispersam dos licores analisados. O coeficiente de variação de Pearson $(\mathrm{CV})$ determinado para cada conjunto está presente na Tabela 1:

Tabela 1 - Coeficiente de variação de Pearson entre o resíduo sulfocáustico e os licores.

\begin{tabular}{ccccc}
\hline Parâmetro analisado & CV RS-LB (\%) & CV RS-LN Fraco (\%) & CV RS-LN Forte (\%) & CV RS-LV (\%) \\
\hline $\mathrm{NaOH}(\mathrm{g} / \mathrm{L})$ & 21,52 & 105,69 & 105,69 & 119,58 \\
$\mathrm{Na}_{2} \mathrm{~S}(\mathrm{~g} / \mathrm{L})$ & 19,99 & 94,84 & 94,84 & 26,09 \\
$\mathrm{Na}_{2} \mathrm{CO}_{3}(\mathrm{~g} / \mathrm{L})$ & 65,67 & 13,85 & 13,85 & 124,18 \\
Álcali efetivo $(\mathrm{g} / \mathrm{L})$ & 21,28 & 103,90 & 103,90 & 75,86 \\
Álcali ativo $(\mathrm{g} / \mathrm{L})$ & 21,10 & 102,61 & 102,61 & 52,32 \\
Álcali total $(\mathrm{g} / \mathrm{L})$ & 25,76 & 92,07 & 92,07 & 27,56 \\
Sulfididade $(\%)$ & 1,14 & 15,13 & 15,13 & 6,54 \\
$\mathrm{pH}$ & 3,32 & 1,05 & 1,05 & 0,50 \\
$\mathrm{Na}_{2} \mathrm{SO}_{4}(\mathrm{~g} / \mathrm{L})$ & $\mathrm{n} / \mathrm{d}$ & $\mathrm{n} / \mathrm{d}$ & $\mathrm{n} / \mathrm{d}$ & 90,65 \\
Sólidos totais $(\%)$ & $\mathrm{n} / \mathrm{d}$ & 21,69 & 69,98 & $\mathrm{n} / \mathrm{d}$ \\
Sólidos suspensos $(\mathrm{g} / \mathrm{L})$ & 16,06 & $\mathrm{n} / \mathrm{d}$ & $\mathrm{n} / \mathrm{d}$ & 122,19 \\
Viscosidade $(\mathrm{mPa} . \mathrm{s})$ & $\mathrm{n} / \mathrm{d}$ & $\mathrm{n} / \mathrm{d}$ & $\mathbf{1 4 0 , 9 4}$ & $\mathrm{n} / \mathrm{d}$ \\
\hline CV médio $(\%)$ & $\mathbf{2 1 , 7 6} \pm \mathbf{1 8 , 5 3}$ & $\mathbf{6 1 , 2 0} \pm \mathbf{4 6 , 3 0}$ & $\mathbf{7 4 , 0 1} \pm \mathbf{4 7 , 5 9}$ & $\mathbf{6 4 , 5 5} \pm \mathbf{4 8 , 5 1}$ \\
\hline
\end{tabular}

Observa-se que as características químicas e físicas do resíduo sulfocáustico se assemelham em sua maioria ao licor branco e se dispersam mais às do licor negro forte. Considerando que as características do licor de cozimento ditam o processo de polpação, que resíduo sulfocáustico é oriundo de petroquímicas, que obteve-se em sua caracterização considerável quantidade de sólidos totais, e que o licor branco deve estar livre de sólidos e de compostos orgânicos, a incorporação do resíduo ao licor branco é inviável.

Em relação ao licor verde, suas características apresentaram alta dispersão das características do resíduo sulfocáustico, principalmente pelo teor de $\mathrm{Na}_{2} \mathrm{CO}_{3}$. O licor verde é rico em $\mathrm{Na}_{2} \mathrm{CO}_{3}$, e a adição do resíduo sulfocáustico a este comprometeria principalmente a etapa de caustificação, etapa em que se recupera o $\mathrm{NaOH}$ necessário ao licor de cozimento, através da reação do $\mathrm{Na}_{2} \mathrm{CO}_{3}$ contido com $\mathrm{Ca}(\mathrm{OH})_{2}$ adicionado.

A dispersão entre o resíduo sulfocáustico e o licor negro forte foi o maior valor encontrado. As diferenças relacionadas ao teor de sólidos totais e a viscosidade foram determinantes para esta alta dispersão. Sendo assim, a adição do resíduo sulfocáustico ao licor negro forte não seria adequada, pois levaria à sua diluição. Considera-se então a alternativa de adição do resíduo sulfocáustico ao licor negro fraco, antes da evaporação.

O resíduo apresentou valores superiores ao licor negro fraco em relação aos sais de sódios e álcalis em geral. Contudo, essas características teoricamente não impedem de adicioná-lo ao licor negro fraco. O resíduo apresentou ainda teor de sólidos totais próximo ao encontrado para o licor negro fraco. Submetendo-os ao processo de evaporação, ao final desta operação, obter-se-ia um licor negro forte rico em matéria orgânica.

Um aspecto muito importante do processo é a adição de $\mathrm{Na}_{2} \mathrm{SO}_{4}$ ao licor negro forte, nas caldeiras de recuperação, objetivando recuperar um dos compostos ativos do licor de cozimento, o $\mathrm{Na}_{2} \mathrm{~S}$, através da redução do sulfato adicionado. $\mathrm{O}$ resíduo sulfocáustico apresentou teor de $\mathrm{Na}_{2} \mathrm{SO}_{4}$, que embora pequeno, permite associar que a possível adição do resíduo ao licor negro pode reduzir a quantidade a ser adquirida e, desta forma conseguir, além do ganho energético com a queima, um ganho econômico, pois além de possuir este químico, já possui um teor considerável de $\mathrm{Na}_{2} \mathrm{~S}$, um dos agentes ativos no licor de cozimento. $\mathrm{O} \mathrm{NaOH}$ contido no resíduo sulfocáustico também pode ser convertido a $\mathrm{Na}_{2} \mathrm{CO}_{3}$ 
- reação que também ocorre na caldeira de recuperação - gerando um licor verde com maior teor de carbonato de sódio - o que aumentaria a eficiência de caustificação - composto este que, na etapa de caustificação, retorna a $\mathrm{NaOH}$, o outro agente ativo no licor de cozimento.

Sendo assim, a etapa mais adequada para a inserção do resíduo sulfocáustico no ciclo de recuperação química dos licores é no estágio que antecede à operação de evaporação.

\section{CONCLUSÕES}

A adição do resíduo sulfocáustico ao licor branco mostrou-se inviável, pois as características do resíduo poderiam prejudicar o processo de deslignificação. O baixo conteúdo de $\mathrm{Na}_{2} \mathrm{CO}_{3}$ no resíduo inviabiliza sua incorporação ao licor verde, dado que este está diretamente ligado à eficiência de caustificação. A baixa viscosidade e o reduzido teor de sólidos totais inviabilizam a adição do resíduo ao licor negro forte, dado que esta adição levaria a diluição do licor concentrado. Concluiu-se que a melhor etapa para adição do resíduo sulfocáustico ao processo Kraft é no estágio que antecede a evaporação, ao licor negro fraco.

Na etapa de evaporação, os mesmos serão concentrados, para então serem queimados na caldeira de recuperação, gerando eletricidade para a fábrica e vapor para o processo. A adição do resíduo sulfocáustico ao licor negro fraco tem potencial de reduzir a aquisição de $\mathrm{Na}_{2} \mathrm{SO}_{4}$, bem como seu conteúdo de $\mathrm{NaOH}$ resultaria em uma maior conversão deste composto em $\mathrm{Na}_{2} \mathrm{CO}_{3}$, etapas ocorridas na caldeira de recuperação. Um maior teor de $\mathrm{Na}_{2} \mathrm{CO}_{3}$ no licor verde aumentaria a conversão deste a $\mathrm{NaOH}$ na etapa de caustificação.

Portanto, conclui-se que o resíduo sulfocáustico possui grande similaridade com os licores do processo Kraft, bem como pode reduzir os custos com químicos de reposição, e beneficiando ainda a petroquímica responsável pela geração deste resíduo, com a redução do volume de efluente gerado e dos custos de tratamento do mesmo.

\section{REFERÊNCIAS}

ALVES, E. D.; PINHEIRO, O. S.; COSTA, A. O. S. da; COSTA JUNIOR, E. F. da. Estudo do processo de obtenção celulose Kraft com ênfase no forno de cal. Rev. Liberato, Novo Hamburgo, v. 16, n. 26, p. 101-220, jul./dez. 2015.

CARDOSO, M.; PASSOS, M. L.; OLIVEIRA, E. D. de. Licor negro de eucalipto Kraft proveniente de indústrias brasileiras: características químicas, físicas e seu processamento na unidade de recuperação. O Papel, São Paulo, v. 67, n. 2, p. 57-83, mai. 2006.

FIGUEIRÊDO, L. S. Modelagem matemática do estado estacionário de um real sistema de caustificação em uma fábrica de celulose. 2009. 71 f. Dissertação (Mestrado em Engenharia Industrial) - Unileste, Coronel Fabriciano, 2009.

ISENMANN, A. F. Química a partir de recursos renováveis. 1. ed. Timóteo: Centro Federal de Educação Tecnológica de Minas Gerais - CEFET-MG, 2012. 105 p.

KETU, J. A. Treatment and disposal of spent caustic at Tema Oil Refinery (TOR). 2013. 70 f. Thesis (Master of Science in Environmental Resources Management) - Kwame Nkrumah University, Kumasi, 2013.

KLOCK, U.; ANDRADE, A. S. de; HERNANDEZ, J. A. Manual didático polpa e papel. 3.ed. Curitiba: Universidade Federal do Paraná, 2013. 118 p.

SIXTA, H.; POTTHAST, A.; KROTSCHEK, A. W. Chemical Pulping Processes. In: SIXTA, H. (Ed.). Handbook of Pulp. Weinheim: Wiley-VCH, 2006. Parte 1, Cap. 4, p. 109-509. 